Article

\title{
Study of a Carbon Nanowall Synthesized on an MWCNT-Based Buffer Layer for Improvement of Electrical Properties
}

\author{
Joouk Kim ${ }^{1}$, Chulmin Park ${ }^{1}$, Hosung Jung ${ }^{1}$, Hyungchul Kim ${ }^{1}$, Seokhun Kwon ${ }^{2}$, \\ Hyeokjoo Choi ${ }^{2}$ (I) and Hyunil Kang ${ }^{2, *}$ \\ 1 Korea Railroad Research Institute, 176 Cheoldobangmulgwan-ro, Uiwang-si 16105, Korea; \\ jookim@krri.re.kr (J.K.); cmpark@krri.re.kr (C.P.); hsjung@krri.re.kr (H.J.); hckim@krri.re.kr (H.K.) \\ 2 Department of Electrical Engineering, Hanbat National University, Daejeon 34158, Korea; \\ kwon1567@naver.com (S.K.); hyukju1210@hanmail.net (H.C.) \\ * Correspondence: hikang@hanbat.ac.kr; Tel.: +82-42-821-1091
}

Received: 29 November 2019; Accepted: 23 December 2019; Published: 25 December 2019

\begin{abstract}
We conducted experiments to improve the electrical properties of the CNW (carbon nanowall), which has lower electrical properties than other carbon allotropes such as graphene and CNT (carbon nanotube), and report the results through this article. The carbon nanowall has an amorphous buffer layer, leading to low electrical properties, and MWCNT (multi-walled carbon nanotube) was used as a buffer layer to improve this issue, and then a CNW was grown on it by CVD (chemical vapor deposition). Then, the content of MWCNT was adjusted to $30 \mu \mathrm{L}, 50 \mu \mathrm{L}$, and $70 \mu \mathrm{L}$ to analyze the electrical properties accordingly. Alteration in carrier concentration, carrier mobility and resistivity were observed as electrical properties. Dramatic changes in electrical properties with MWCNT content were identified. The ohmic contact state between the MWCNT-based buffer layer and the CNW was investigated by analysis of the I-V and I-R characteristics and the electrical stability according to the linearity of the curve.
\end{abstract}

Keywords: carbon nanowall; Raman spectroscopy; hall measurement system; carrier concentration; carrier mobility; electrical characteristic

\section{Introduction}

The new industries in various fields are in the spotlight with the introduction of the new industrial era. One of them is the next generation material development industry. Carbon material is greatly popular and a promising material among existing materials, and is able to be used in various fields such as displays, electron application devices, wearable devices, batteries and renewable energy [1-4]. Typical carbon materials include graphene, carbon nanotube (CNT), diamond-like carbon (DLC), carbon black and fullerene. Graphene and CNTs have outstanding electrical, mechanical, thermal, and flexibility properties, and with various studies that have been conducted, they have reached the step before commercialization of the application industry. In addition to these, the carbon nanowall (CNW) is a material that has unlimited potential. CNW has many advantages with carbon allotrope, like the materials mentioned above. $\mathrm{CNW}$, with its multi-layer graphenes standing perpendicular to the substrate, has the largest surface area of any carbon allotrope [5]. CNW is actively used in application sensors because the large surface area can be applied as a great advantage in application devices such as sensors. Especially, in the case of a sensor adopted with the electrochemical mechanism, it can be a great advantage [6]. However, CNW has many porous-like air gaps on its surface, so its electrical properties are poor compared to graphene and CNT. CNW also has a buffer layer for nucleation 
and graphene layer growth. This buffer layer is an amorphous carbon layer [7]. The material, having a general amorphous structure, does not have remarkable electrical properties because of the non-crystallization and non-orientation. In order to alleviate these disadvantages, various experiments have been conducted to improve the surface and electrical properties of CNW. Improvements in properties through pre-treatment and post-treatment are typical, and there are various methods such as utilizing metal-based particles or doping gaseous gases.

In this experiment, the electrical properties were improved by using MWCNT (multi-walled carbon nanotube) as the buffer layer of a carbon nanowall. After forming a buffer layer with different CNT contents, CNW was synthesized by chemical vapor deposition. Thereafter, a lot of electrical characteristics were analyzed and compared with the general CNW.

\section{Experimental}

Si wafers with (111) orientation were soaked in acetone and ultrasonically cleaned for $15 \mathrm{~min}$. After, acetone-treated Si wafers were immediately put into methanol and cleaned for $10 \mathrm{~min}$ to blow off the surface acetone components. The final washing and drying were performed using DI pure water and N2 gas. Then, MWCNT dispersed in water (US Research Nanomaterials, Inc., Houston, TX, USA, 4 science) having an outside diameter of 5-15 nm and length of $50 \mu \mathrm{m}$ was used to form a MWCNT-based buffer layer on the prepared Si wafer, and detailed specifications of MWCNT are shown in Table 1. The amount of MWCNTs used for the formation of the buffer layer was adjusted to 30, 50, and $70 \mu \mathrm{L}$, and dropped on a Si wafer, followed by drying at $50^{\circ} \mathrm{C}$ for $1 \mathrm{~h}$ using a hot plate. The CNW was grown on a MWCNT-based buffer layer using microwave PECVD. The growth conditions of CNW are $1300 \mathrm{~W}$ (microwave power), growth time $(10 \mathrm{~min}), 700{ }^{\circ} \mathrm{C}$ (substrate temperature), gas ratio $\left(\mathrm{H}_{2}\right.$ : $25 \mathrm{sccm}$ and $\mathrm{CH}_{4}: 50 \mathrm{sccm}$ ), $2.5 \times 10^{-2}$ torr (working pressure). The various analyses were operated to investigate the characteristics of CNW grown on a MWCNT-based buffer layer. FE-SEM (field emission scanning electron microscopy, Hitachi-S4800) was operated for surface and cross section analysis under acceleration voltage (10 15 kV), emission current (8600 nA), and lens mode (high). Raman spectra (FEX MRA10, NOST) analysis was performed to examine the successful growth of CNW and structural changes caused by the MWCNT-based buffer layer under conditions such as excitation laser wavelength $(\sim 531 \mathrm{~nm})$, excitation laser power $(\sim 0.30 \mathrm{~mW})$, objective lens $(50 \times)$ and spectral resolution (1.87-2.89 $\left.\mathrm{cm}^{-1}\right)$. The hall measurement system (HMS-3000, Ecopia) analysis was conducted to observe the electrical characteristics such as carrier concentration, carrier mobility, resistivity, I-V characteristics, and I-R characteristics. Then, the applied current was $1 \mathrm{~mA}$ under magnetic field $(0.550 \mathrm{~T})$, and measurement number and temperature were set to 1000 times and room temperature, respectively. The overall experimental procedure is shown in Figure 1.

Table 1. Specification of MWCNT (multi-walled carbon nanotube).

\begin{tabular}{cccccc}
\hline Purity & Diameter & Length & Ash & $\begin{array}{c}\text { Electrical } \\
\text { Conductivity }\end{array}$ & Density \\
\hline $\begin{array}{c}95 \mathrm{wt} \%(\text { CNT_carbon nanotube) } \\
95 \mathrm{wt} \% \text { (Carbon) }\end{array}$ & $\begin{array}{c}\text { Out: } 5-15 \mathrm{~nm} \\
\text { In: } 3-5 \mathrm{~nm}\end{array}$ & $50 \mu \mathrm{m}$ & $1.5 \mathrm{t} \%$ & $100 \mathrm{~s} / \mathrm{cm}$ & $\begin{array}{c}\text { Tap: } 0.27 \mathrm{~g} \\
\text { True: } \sim 2.1 \mathrm{~g}\end{array}$ \\
\hline
\end{tabular}


(a)

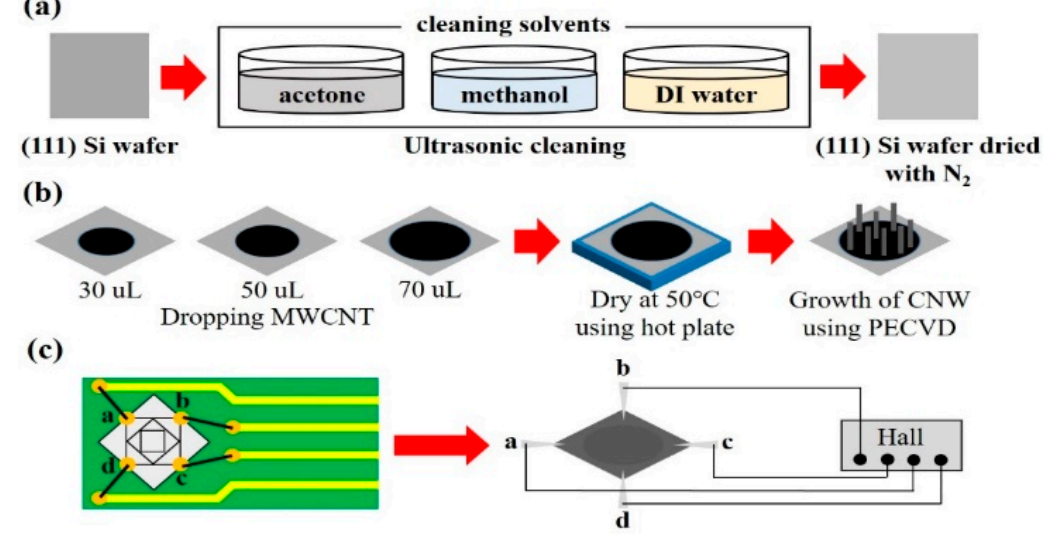

Figure 1. Schematic of experiments: (a) cleaning of Si wafer substrate; (b) synthesis of CNW (carbon nanowall) on MWCNT-based buffer layer; (c) measurement of electrical properties of samples.

\section{Results}

Figure 2 shows the image of FE-SEM of pristine CNW and MWCNT/CNW. The pristine CNW showed tight cross-sections with typical CNW morphology with various nano-porous surface (Figure 2a). On the other hand, all samples where MWCNT was used as the buffer layer exhibited thick buffer layers compared to the pristine CNW (Figure 2b-d). The thickness of the MWCNT-based buffer layer with $30,50,70 \mu \mathrm{L}$ was $7.8,8.3$, and $8.7 \mu \mathrm{m}$, respectively. As the concentration increased, the buffer layer tended to become thicker. The height of CNW examined in all samples was $1.8 \sim 2.0 \mu \mathrm{m}$. In surface FE-SEM, only the arrangement of nanowalls was different with no-change in shape and density. These no-change of the CNW surface is different from the carbon nanowalls grown on different substrates [8].
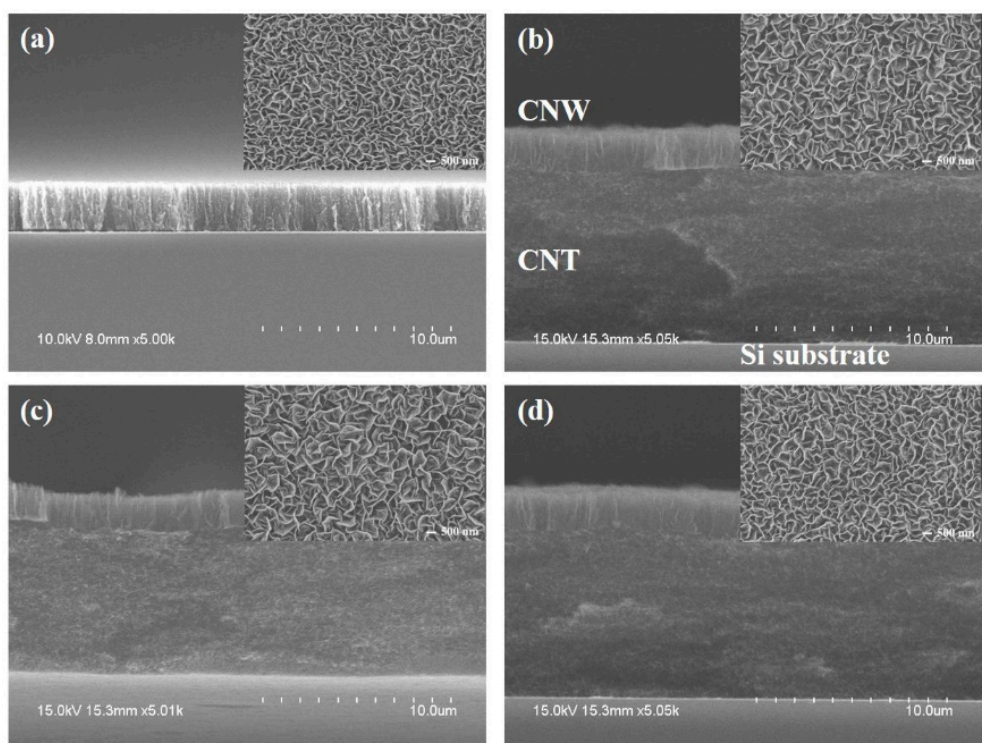

Figure 2. The image of surface (inset) and cross section FE-SEM: (a) pristine MWCNT (b) CNT $(30 \mu \mathrm{L}) / \mathrm{CNW}$; (c) MWCNT $(50 \mu \mathrm{L}) / \mathrm{CNW}$; (d) MWCNT $(70 \mu \mathrm{L}) / \mathrm{CNW}$.

Figure 3 shows the Raman spectra results of all samples. In Figure 3a, three peaks that can be commonly found in carbon materials were identified. Three common peaks are D peak $\left(1350 \mathrm{~cm}^{-1}\right)$, G peak $\left(1580 \mathrm{~cm}^{-1}\right)$, and 2D peak $\left(2700 \mathrm{~cm}^{-1}\right)$. The D peak near $1350 \mathrm{~cm}^{-1}$ can confirm the degree of defects due to the alignment of the carbon crystals. The high $\mathrm{D}$ peaks were observed in all samples, and these characteristics can be easily investigated in CNT and CNW. G peaks near $1580 \mathrm{~cm}^{-1}$ are common in graphite-based carbon materials. The higher the intensity of the $\mathrm{G}$ peak, the better the 
graphitization and the better the carbon material. Then, the D peak occurred at $1620 \mathrm{~cm}^{-1}$ was able to be examined only in CNW. This peak is mainly discovered at the edge of graphene. CNW is vertically grown with multiple graphene layer on a substrate, and the top of CNW may be opened to the edge of graphene. In addition, CNW has a large number of carbon branches, and the peak of D peak can be occurred because the end of this branch can have the shape of a graphene edge. On the other hand, in the case of CNT, single or multilayer graphene layers are curled and the edge of the graphene cannot be identified, so D peak does not appear. The 2D peak of $2700 \mathrm{~cm}^{-1}$ appeared in all samples, and as the $\mathrm{I}_{2 \mathrm{D}} / \mathrm{I}_{\mathrm{G}}$ ratio of 0.5 or less was confirmed, it may imply that all samples had a multilayer graphene structure. Figure $3 b$ shows the $\mathrm{I}_{\mathrm{D}} / \mathrm{I}_{\mathrm{G}}$ ratio of all samples. The $\mathrm{I}_{\mathrm{D}} / \mathrm{I}_{\mathrm{G}}$ ratio numerically shows the carbon material defects, and the lower the ratio, the better the quality of the carbon material. All CNW specimens had a higher $\mathrm{I}_{\mathrm{D}} / \mathrm{I}_{\mathrm{G}}$ ratio than $\mathrm{CNT}$. With this phenomenon, the mechanism of growth of carbon nanowalls began with a defect by mismatch of carbon onions formed on the amorphous carbon buffer layer [9]. When MWCNT is used as a buffer layer, carbon radicals by CVD can induce C-C bonds with MWCNTs, not substrates, thereby forming graphitic layers more than amorphous carbon. It may be a cause that can lead to improved electrical properties. In addition, carbon nanowalls usually change in structure and properties depending on the substrate [10-12]. In our research, it is suggested that the structure of all specimens was unchanged because it was a synthesis reaction between the carbon-based substrate and carbon radical, not a reaction to the Si-substrate and the carbon radical, and it caused a non-variation of Raman spectra results.
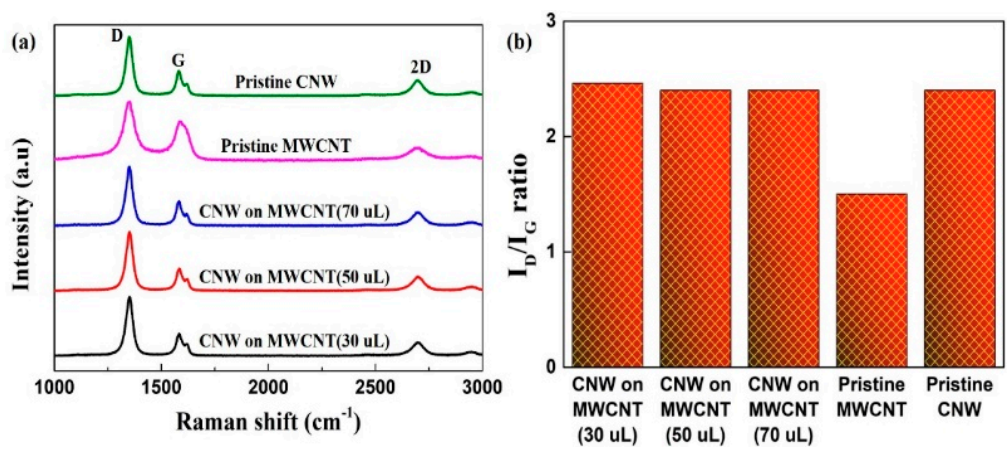

Figure 3. Structural properties results of all samples: (a) Raman spectra; (b) $\mathrm{I}_{\mathrm{D}} / \mathrm{I}_{\mathrm{G}}$ ratio.

Figure 4 shows the electrical properties of carbon nanowalls grown on MWCNT-based buffer layers. In the Raman spectra, CNW grown on the MWCNT-based buffer layer was not significantly different from normal CNW. However, interesting results were found in the electrical properties results. First, in the case of carrier concentration, an improvement was observed with the increase of the CNT content forming the MWCNT-based buffer layer compared to the pristine CNW. The carbon buffer layer, which plays an important role in the general CNW nucleation and growth mechanism, is an amorphous carbon layer, so excellent electrical properties cannot be expected. While the MWCNT-based buffer layer has better electrical properties than the amorphous carbon layer, it is considered to contribute to the improvement of carrier concentration because it contains many carriers as a semiconductor impurity concept. Second, the carrier mobility was found to be inversely proportional to the carrier concentration. This phenomenon may be caused by alignment and defects in the carbon lattice. The MWCNT-based buffer layer underneath the CNW also has a high carbon lattice disorder similar to that of the CNW, which can significantly affect carrier mobility. The resistivity characteristic was analyzed to decrease with the increase of CNT content. This is because the carrier concentration is dominant in resistive properties, and the increase in the number of carriers by the CNT is expected to lead to a decrease in the resistivity. For this reason, samples containing $70 \mu \mathrm{L}$ of CNTs showed the outstanding carrier concentration and resistivity. 


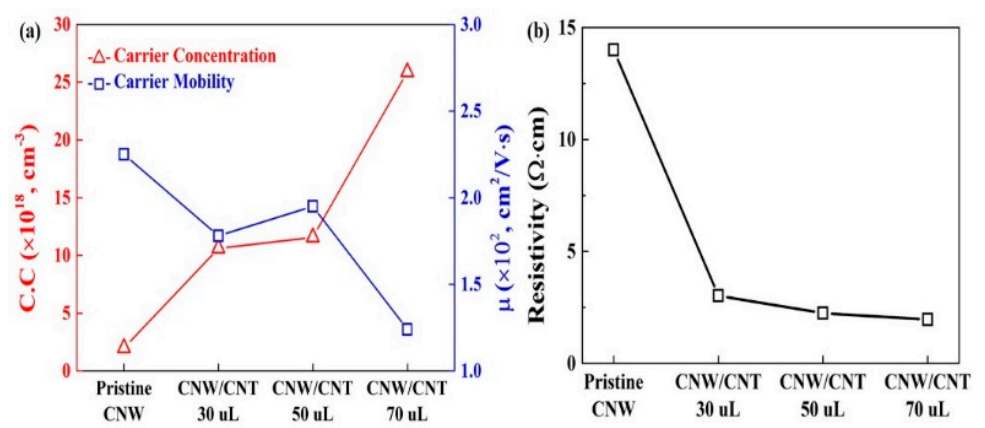

Figure 4. Electrical properties of all samples: (a) carrier concentration and carrier mobility; (b) resistivity of all samples.

Figure 5 shows the I-V characteristics of pristine CNW, MWCNT $(30 \mu \mathrm{L}) / \mathrm{CNW}$, MWCNT $(50 \mu \mathrm{L}) / \mathrm{CNW}$ and MWCNT $(70 \mu \mathrm{L}) / \mathrm{CNW}$ samples. As shown in Figure 1c, four probe tips were contacted at the edge of a sample having a size of $1 \mathrm{~cm} \times 1 \mathrm{~cm}$ to apply a voltage of $-1 \mathrm{mV}$ to $+1 \mathrm{mV}$. The slopes of the curves were different for all samples, but a linear I-V curve was observed. Since the slope of the curve represents the measured resistance value, it does not have much significance for the stability of the electrical characteristics. However, linearity is the most important factor because it implies that a constant current and resistance are maintained whenever the applied voltage changes. The V-I characteristics appear that all samples are electrically stable, which is due to ohmic contacts, not schottky contacts. Figure 6 shows the change in resistance with the applied current as an I-R characteristic and average $\Delta \mathrm{R}$ with percentage. An important factor of the I-R characteristics is linearity. This is because the resistance does not change even when the current changes, which means that it is electrically stable. In pristine CNW, parabolic curves were observed in all sections, indicating that there is a change in resistance due to current changes, suggesting this sample is not stable. In the CNW sample formed on the MWCNT-based buffer layer, the improved linearity occurred. As the CNT content was increased, the linearity was dramatically improved. As shown in Figure $6 \mathrm{~d}$, the I-R characteristics with a CNT content of $70 \mu \mathrm{L}$ show highly improved linearity. However, in Figure 6b,c, partial nonlinearities have been identified. It is noted that these phenomena may be caused by structural changes in CNTs and CNWs, and defects caused by various factors.
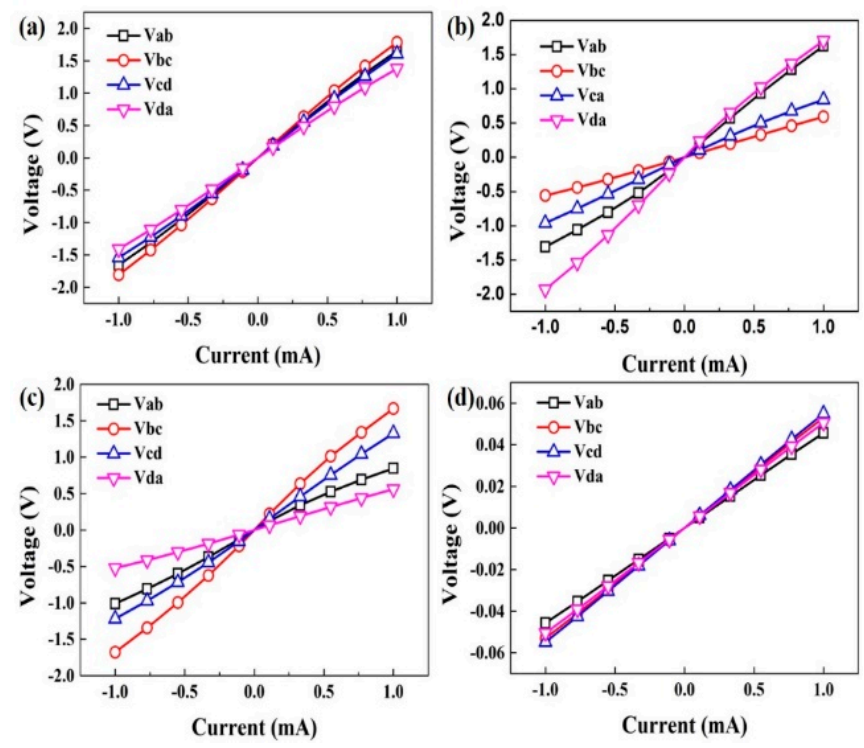

Figure 5. Electrical stability with I-V curve of all samples: (a) pristine CNW; (b) MWCNT (30 $\mu \mathrm{L}) / \mathrm{CNW}$; (c) MWCNT $(50 \mu \mathrm{L}) / \mathrm{CNW}$; (d) MWCNT $(70 \mu \mathrm{L}) / \mathrm{CNW}$. 

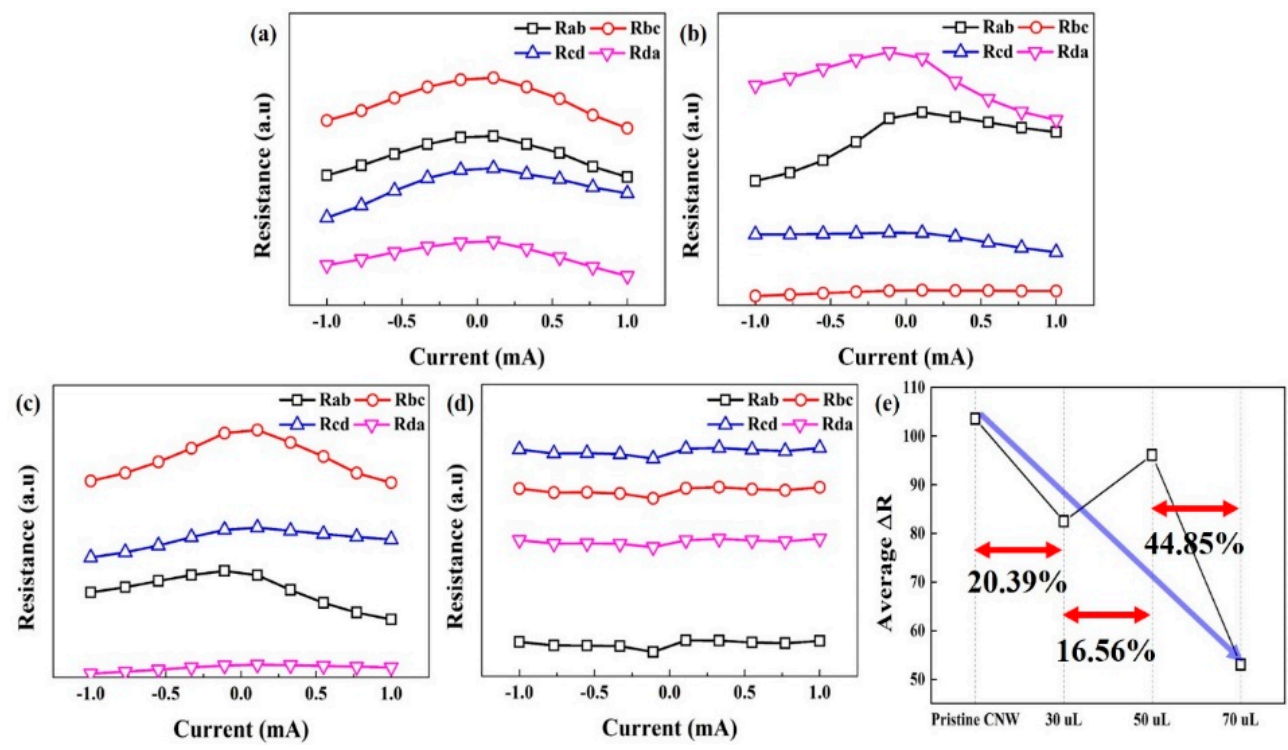

Figure 6. Electrical stability with I-R curve of all samples: (a) pristine CNW; (b) MWCNT (30 $\mu \mathrm{L}) / \mathrm{CNW}$; (c) MWCNT $(50 \mu \mathrm{L}) / \mathrm{CNW}$; (d) MWCNT $(70 \mu \mathrm{L}) / \mathrm{CNW}$; (e) average $\Delta \mathrm{R}$ with percentage.

In addition, stability test was conducted by obtaining the average $\Delta \mathrm{R}$ at all points of each sample, and it means the better stability with lower $\Delta \mathrm{R}$. As expected, the pristine CNW showed the largest $\Delta \mathrm{R}$ because the amorphous carbon was formed into the buffer layer, causing the charge transfer to be inconsistent. The sample with $30 \mu \mathrm{L}$ of a MWCNT-based buffer layer showed a $20.39 \%$ decrease compared to pristine CNW. The samples using MWCNT with the highest concentration of $70 \mu \mathrm{L}$ showed the lowest variation and the outstanding stability. It is noted that the sudden increase in the sample with $50 \mu \mathrm{L}$ may be due to surface contamination during drying of the MWCNT after dropping MWCNT onto the Si substrate. Additionally, when measuring the hall measurement, such a phenomenon may occur because of scratches on the surface.

\section{Conclusions}

Carbon nanowalls show low electrical properties due to the amorphous buffer layer. In order to improve this portion, the MWCNT was used as a buffer layer, and the structural and electrical properties were studied. The structural characteristics by Raman spectra were not significantly different from those of general CNWs. This is because the Raman spectra only analyzes the edge part of a thin film surface, not bulk analysis, suggesting that the CNW has been successfully grown on MWCNT-based buffer layers. The dramatic changes were identified in the electrical characterization. As the CNT content increased, the carrier concentration was improved and the resistivity was dramatically decreased. In the I-V characteristics, all samples showed linearity, indicating that they were electrically stable, and a successful ohmic contact could be expected. CNW samples with MWCNT-based buffer layers showed improvement of I-R linearity compared with pristine $\mathrm{CNW}$, and samples containing $70 \mu \mathrm{L}$ of CNTs showed perfect straight lines and perfect stable properties. CNW is a carbon material which has characteristics that differ greatly by pre-treatment, unlike graphene and CNT. In this study, electrical characteristics of CNWs were improved by pre-treatment of MWCNT-based buffer layers. As such, the electrical characteristic conversion method, according to the pre-treatment, is expected to have a high influence on various devices such as biosensors and gas sensors, which will be used in the future. 
Author Contributions: Conceptualization, J.K. and C.P.; methodology, H.J., H.K. and H.C.; formal analysis H.K. and S.K.; software, S.K.; writing, J.K.; supervision, H.K. All authors have read and agreed to the published version of the manuscript.

Acknowledgments: This research was supported by a grant from R\&D Program of the Korea Railroad Research Institute, Republic of Korea and was also supported by the Korea Institute of Energy Technology Evaluation and Planning (KETEP) and the Ministry of Trade, Industry \& Energy (MOTIE) of the Republic of Korea (No. 20184030201900).

Conflicts of Interest: The authors declare no conflict of interest.

\section{References}

1. Zhang, P.; Qiao, Z.A.; Dai, S. Recent advances in carbon nanospheres: Synthetic routes and applications. Chem. Commun. 2015, 51, 9246-9256. [CrossRef]

2. Han, X.; Han, P.; Yao, J.; Zhang, S.; Cao, X.; Xiong, J.; Zhang, J.; Cui, G. Nitrogen-doped carbonized polyimide microsphere as a novel anode material for high performance lithium ion capacitors. Electrochim. Acta 2016, 196, 603-610. [CrossRef]

3. Zarrin, H.; Sy, S.; Fu, J.; Jiang, G.; Kang, K.; Jun, Y.S.; Yu, A.; Fowler, M.; Chen, Z. Molecular Functionalization of Graphene Oxide for Next-Generation Wearable Electronics. ACS Appl. Mater. Interfaces 2016, 8, 25428-25437. [CrossRef]

4. Lou, Z.; Chen, S.; Wang, L.; Jiang, K.; Shen, G. An ultra-sensitive and rapid response speed graphene pressure sensors for electronic skin and health monitoring. Nano Energy 2016, 23, 7-14. [CrossRef]

5. Wang, H.; Gao, E.; Liu, P.; Zhou, D.; Geng, D.; Xue, X.; Wang, L.; Jiang, K.; Xu, Z.; Yu, G. Facile growth of vertically-aligned graphene nanosheets via thermal CVD: The experimental and theoretical investigations. Carbon 2017, 121, 1-9. [CrossRef]

6. Komarova, N.S.; Krivenko, A.G.; Stenina, E.V.; Sviridova, L.N.; Mironovich, K.V.; Shulga, Y.M.; Krivchenko, V.A. Enhancement of the Carbon Nanowall Film Capacitance. Electron Transfer Kinetics on Functionalized Surfaces. Langmuir 2015, 31, 7129-7137. [CrossRef] [PubMed]

7. Zhao, J.; Shaygan, M.; Eckert, J.; Meyyappan, M.; Rummeli, M.H. A Growth Mechanism for Free-Standing Vertical Graphene. Nano Lett. 2014, 14, 3064-3071. [CrossRef] [PubMed]

8. Davami, K.; Shaygan, M.; Kheirabi, N.; Zhao, J.; Kovalenko, D.A.; Rummeli, M.H.; Opitz, R.; Cuniberti, G.; Lee, J.; Meyyappan, M. Synthesis and characterization of carbon nanowalls of different substrate by radio frequency plasma enhanced chemical vapor deposition. Carbon 2014, 72, 372-380. [CrossRef]

9. Ghosh, S.; Ganesan, K.; Polaki, S.R.; Mathews, T.; Dhara, S.; Kamruddin, M.; Tyagi, A.K. Influence of substrate on nucleation and growth of vertical graphene nanosheets. Appl. Surf. Sci. 2015, 349, 576-581. [CrossRef]

10. Zhou, H.; Liu, D.; Luo, F.; Luo, B.; Tian, Y.; Chen, D.; Shen, C. Preparation of graphene nanowalls on nickel foam as supercapacitor electrodes. Micro Nano Lett. 2018, 13, 842-844. [CrossRef]

11. Fang, J.; Levchenko, I.; van der laan, T.; Kumar, S.; Ostrikov, K. Multipurpose nanoporous alumina-carbon nanowall bi-dimensional nano-hybrid platform via catalyzed and catalyst-free plasma CVD. Carbon 2014, 78, 627-632. [CrossRef]

12. Dinh, T.M.; Achour, A.; Vizireanu, S.; Dinescu, G.; Nistor, L.; Armstrong, K.; Guay, D.; Pech, D. Hydrous $\mathrm{RuO}_{2}$ /carbon nanowalls hierarchical structures for all-solid-state ultrahigh-energy-density micro-supercapacitors. Nano Energy 2014, 10, 288-294. [CrossRef]

(C) 2019 by the authors. Licensee MDPI, Basel, Switzerland. This article is an open access article distributed under the terms and conditions of the Creative Commons Attribution (CC BY) license (http://creativecommons.org/licenses/by/4.0/). 